\title{
Rückblick auf ein erfolgreiches Jahr
}

Noch wenige Tage, dann ist das Jahr 2008 Vergangenheit genau der richtige Zeitpunkt, um Rückschau auf die für die FMH und die Ärzteschaft wichtigsten Ereignisse zu halten.

Das Jahr begann ziemlich heftig: Kaum waren die neuen Führungsmodelle der FMH beschlossen und in Umsetzung begriffen, galt es, den als parlamentarischen Gegenvorschlag ausgestalteten neuen Art. 117a der Bundesverfassung zu bekämpfen. Dies gelang, indem die FMH

\section{Der Erfolg vom 1. Juni hat der Ärzteschaft im politischen Betrieb klar mehr Einfluss verschafft}

ein Komitee der blauen Berufe gründete und mit diesen Verbündeten eine wirkungsvolle Kampagne unter dem Titel «Nein zum Kassendiktat!» führte. Mit weiteren Allianzen, insbesondere mit den Kantonen, konnten wir dann am 1. Juni einen schon fast historischen Sieg erringen: Das Schweizer Volk verwarf die parlamentarische Vorlage wuchtig mit beinahe $70 \%$ der Stimmenden und lehnte damit die Aufhebung des Vertragszwangs und eine damit verbundene unerwünschte Machtverschiebung zu den Kassen ab.

Dieser klare Sieg stärkte die Position der Ärzteschaft bei anderen für sie wichtigen politischen Themen wie Zulassungsstopp und Nachfolgeregelung, Einfluss auf die ärztlichen Weiter- und Fortbildung, Tarifsenkungen bei Praxislabor und direkter Medikamentenabgabe sowie neue Versicherungsmodelle wie die duale Grundversicherung. Diese Schlachten sind zwar allesamt noch nicht geschlagen, aber der Erfolg vom 1. Juni hat der Ärzteschaft im politischen Betrieb klar mehr Einfluss verschafft. Getreu nach der alten und neuen strategischen Zielsetzung: «Keine Gesundheitspolitik ohne die FMH!»
Der Erfolg vom 1. Juni hat noch andere positive Auswirkungen: Die FMH konnte Allianzen innerhalb der blauen Berufe austesten und kann diese nun nach Bedarf jederzeit rasch aktivieren. Auch die Zusammenarbeit mit der GDK hat die FMH vertieft - was sich bei aktuellen und künftigen Fragestellungen insbesondere bei der Versorgungsplanung möglicherweise als sehr wertvoll erweisen wird.

Verbandsintern ist die Gesamterneuerungswahl des Zentralvorstandes von Ende Mai und dessen gleichzeitige Reduktion von 11 auf 9 Mitglieder zu erwähnen, die - wie sich bereits nach den ersten Monaten zeigt - zu der angestrebten Effizienzsteigerung geführt hat. Als eine seiner wichtigsten Aufgaben hat der neue Zentralvorstand die politischen und strategischen Zielsetzungen für die Legislatur 2008-2012 erarbeitet, die die Ärztekammer vor einigen Tagen genehmigt hat. Eine gute Grundlage für den Start ins nächste Jahr.

\section{Der neue Zentralvorstand hat die politischen und strategischen Zielsetzungen für die Legislatur 2008-2012 erarbeitet}

Nach diesem ereignis- und arbeitsreichen Jahr wünschen wir Ihnen frohe und erholsame Festtage, damit wir mit Ihrer Unterstützung auch im nächsten Jahr alle Angriffe auf unser gut funktionierendes Gesundheitswesen weiterhin erfolgreich parieren können.

Daniel Herzog, Generalsekretär FMH 